\title{
SIGNIFICANCE OF INTENSITY OF SWEDISH MASSAGE COURSE IN IMPROVEMENT OF HEALTH STATUS
}

\author{
Una Veseta \\ Red Cross Medical College of Riga Stradiņš University, Latvia \\ Antra Gulbe \\ Red Cross Medical College of Riga Stradiņš University, Latvia \\ Oskars Onzevs \\ Red Cross Medical College of Riga Stradiņš University, Latvia
}

\begin{abstract}
The aim of the study is to compare the immediate and cumulative effects of Swedish massage on the physiological, emotional, and biochemical factors of healthy individuals, as well as the sustainability of the effects depending on the intensity of the course.The study subjects were 30 healthy young women. The subjects were randomly assigned into two groups. Subjects got in total ten Swedish back massages: twice and once a week. At the research the complete blood count was performed, salivary cortisol level, total antioxidant capacity were checked with the resources of laboratory "E. Gulbja Laboratorija" (www.egl.lv) Emotions were tested by using the FaceReader 6.0 $0^{\mathrm{TM}}$. Body composition analysis was performed by using Tanita SC 330, blood pressure and heart rate were also measured (Omron M7-IT). The collected data was analyzed by using methods of mathematical statistics. Regression testing was performed by using the Fisher criterion. Analysis of the obtained data allows us to observe positive trends in Swedish massage to the improvement of health status. Differences in some summary results of massage effects were identified.
\end{abstract}

Keywords: course intensity, healthy individuals, health status, Swedish massage.

\section{Introduction}

Massage therapy is a purposeful, widely recognized medical technology, which applies dosed mechanical influence to the soft tissues of the human body skin, tendons, ligaments, fascia, and muscles (Fritz, 2014). The effect produced by massage in the human's organism is a complicated physiological process, which involves numerous systems and organs, but the leading role belongs to the nervous system (Fritz, 2014; Moraska, Pollini, Boulanger, Brooks, \& Teitlebaum, 2010).

Despite the growing popularity of massage, most of the published research materials have very cautiously formulated conclusions: massage may reduce pain, stress, symptoms of depression and insomnia, it can improve certain parameters 
Veseta et al., 2020. Significance of Intensity of Swedish Massage Course in Improvement of Health Status

of immunity. There isn't much research that has addressed the issue of how massage helps, i.e. it's mechanisms of action, instead of whether it helps. Research on the immediate effects of massage and the effects of repeated influence of massage on the body is lacking and the significance of the "dosage" of influence (interval between sessions) is a completely new research direction (Moyer, Rounds, \& Hannum, 2004).

The aim of the study is to compare the immediate and cumulative effects of Swedish massage on the physiological, emotional, and biochemical factors of healthy individuals, as well as the sustainability of the effects depending on the intensity of the course.

\section{Literature review}

In the most of researches, massage is done twice a week (Fielda, 2016). According to the results of Fateme, Mojtaba, \& Hojatollah research, it is recommended that massage, as a valuable non-invasive method, is used for nurses in intensive care units to reduce their stress, promote mental health and prevent the decrease in quality of nursing work life. Swedish massage was performed on participants of the experimental group for 25 min in each session, twice a week for four weeks (Fateme, Mojtaba, \& Hojatollah, 2015).

The findings of another research indicate that massage therapy enhances positive well-being and reduces stress perception among community-dwelling older adults. Participants ( $\mathrm{n}=54$ ) got 50 min massage or guided relaxation sessions twice weekly for four weeks. The massage included Swedish, neuromuscular and myofascial techniques (Sharpe, Williams, Granner, \& Hussey, 2007).

The effect of a single Swedish massage session (45 min) on the oxytocin level of a healthy adult, hypothalamic-pituitary-adrenal activity and improvement in immunity was researched at the University of Atlanta in 2009. The study did not confirm a significant increase in oxytocin levels, but a minor decrease in cortisol level was observed and the overall conclusion was made that a single massage session cannot significantly reduce the factors that characterize stress (Rapaport, Schettler, \& Breese, 2010). Therefore, the researchers at the University of Atlanta conducted another study in 2011, where the effect of a 5week massage course (once or twice a week), where the factors characterizing stress and immunity of a healthy adult were researched. The researchers concluded that the cumulative effect of massage may differ, depending on the intensity of massage sessions, as well as stated that further research is required (Rapaport, Schettler, \& Breese, 2012).

Cortisol has been indicated as a stress characterizing factor in several studies on the effect of massage (Bunker et al., 2003; Dickerson \& Kemeny, 2004; Field, Hernandez-Reif, Diego, Schanberg, \& Kuhn, 2005). For example, Field, et al. 
(1996), twenty-six adults got a chair massage and 24 control group adults were asked to relax in the massage chair for 15 minutes, two times per week for five weeks. Group by repeated measures and post hoc analyses revealed the following: 1) frontal delta power increased for both groups, suggesting relaxation; 2) the massage group showed decreased frontal alpha and beta power (suggesting enhanced alertness); while the control group showed increased alpha and beta power; 3) the massage group showed increased speed and accuracy on math computations while the control group did not change; 4) anxiety levels were lower following the massage, but not the control sessions, although mood state was less depressed following both - the massage and control sessions; 5) salivary cortisol levels after the massage were lower only for one day, but not after the control sessions; 6) at the end of the five-week period depression scores were lower for both groups, but job stress score were lower only for the massage group (Field et al., 1996).

Seven weeks course massage therapy consisted of a 20 min massage therapy session offered daily to patients (a young adult psychiatric inpatient) during the period of hospitalization. There was a significant reduction in self-reported anxiety $(\mathrm{p}<0.001)$, resting heart rate $(\mathrm{p}<0.05)$ and cortisol levels $(\mathrm{p}<0.05)$ immediately following the initial and final massage therapy sessions (Garner et al., 2008).

Two similar design studies in Givi (2013) and Moeini, Givi, Ghasempour, \& Sadeghi (2011) shows the effects of massage on blood pressure (BP). It was performed on women with pre-hypertension. The study consisted of two groups test group (25 patients) getting 10 sessions of Swedish massage (10-15 min) three times a week, and the control group (25 patients), which was offered a relaxation in the same environment without getting any massage. The results indicated that mean systolic and diastolic blood pressure in the massage group was significantly lower in comparison with the control group $(\mathrm{p}<0.001)$. Evaluation of durability of the massage effects on BP also indicated, that even 72 hours after finishing the study there was still a significant difference between the test and control groups in systolic and diastolic BP $(p<0.001)$. Findings of the study indicated that massage therapy was a safe, effective, applicable and cost-effective intervention in controlling BP of the pre-hypertension women and it can be used in the health care centers and even at home (Givi, 2013; Moeini, Givi, Ghasempour, \& Sadeghi, 2011).

A study conducted in Japan included 12 elderly people requiring long-term care, who were randomly divided into two groups (A and B). Group A got hand massage and group B got foot massage -for 15 min each. After one week, group A got foot massage and group B got hand massage - for 15 min each. Results showed that the degree to which participants felt pleasant, relaxed and refreshed was high after both kinds of massage. This study suggests that hand and foot 
Veseta et al., 2020. Significance of Intensity of Swedish Massage Course in Improvement of Health Status

massage modulate psychological factors in elderly people requiring long-term care and can be used more regularly to support the well-being of elderly people (Nakano et al., 2019).

In general, the findings of studies carried out so far have shown that massage effectively improves health and well-being, both in virtually healthy subjects and in people with different diseases. Massage has been performed twice a week in most of studies. The effect of massage on different health parameters has been demonstrated, but did not find any studies of the immediate and cumulative effects of massage and the residual effects of course exposure depending on the intensity of the massage course.

\section{Methodology}

This was a 5 and 10 weeks randomized open-type comparative study that compared the immediate and cumulative effects of Swedish massage to the physiological, emotional and biochemical factors of healthy individuals, as well as the sustainability of massage impact depending on the intensity of the course.

The study was conducted the Red Cross Medical College of Riga Stradiņs University (hereinafter - RSU RCMC) scientific activities development project "The importance of classical massage course intensity in improving health status" No 1.), which took place in the for ten months in accordance with generally accepted standards of good scientific practice and ethical principles provided by the Helsinki Declaration. The protocol was reviewed and approved RSU RCMC ethical committee. Before being included in the study, each participant signed the informed consent to participate in the study.

The main inclusion criteria of the study participants were: a physically and psychologically healthy person aged 18 to 35 who did not smoke, has not taken within the last six months more than a glass of wine or its equivalent (another alcohol) per day, has not used drugs, has not worked night shifts, denied pregnancy, failed to follow a special diet, the body mass index (BMI) was within the normal range of 18.5 to 24.9 and the results of a full blood test were consistent with the generally accepted normal values of a healthy person.

The main exclusion criteria were:

- $\quad$ serious medical problems with recent history of medical interventions;

- $\quad$ participant prepares to follow some health-affecting program during the study;

- risk of not completing the study (it was previously known that participant will not be able to attend all massage and test sessions). 


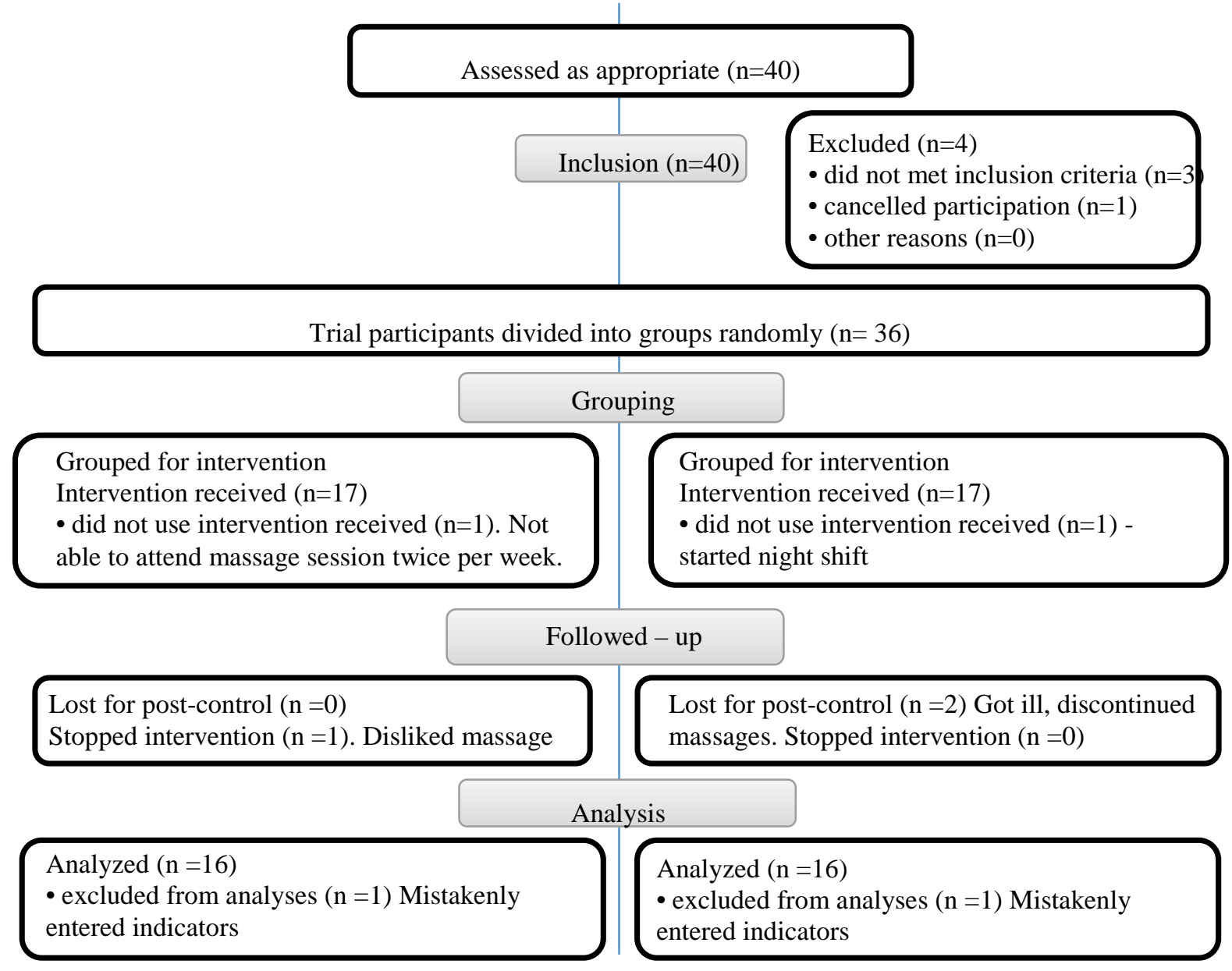

Figure 1 Study Flow Scheme

Following the first anthropometric and laboratory assessments, the participants were divided into groups (randomly) using the drawing lots (Figure 1). Group A members were massaged twice a week, i.e., they had a fiveweek course, while group B members were massaged once a week and had a 10-week course.

Methods. The weight was determined using professionally calibrated scales and the analyser Tanita SC 330 (N 11030184 TANITA 545 N 510426) to the nearest $100 \mathrm{~g}$. The participants were wearing lightweight clothing, no shoes.

The clinical blood test was conducted to assess the actual health status of the study participants: (Hb, WBC, RBC, HT, PLT) + WBC differential + ESR (capillary photometry method). The results of the analysis showed the results of the patient's blood composition and generally recognized reference ranges for the healthy person.

Full blood counts were detected, cortisol levels in saliva and total antioxidant capacity measured at the beginning of the study, after the fifth massage, after the last massage and two weeks after the last massage. The levels of cortisol in the 
Veseta et al., 2020. Significance of Intensity of Swedish Massage Course in Improvement of Health Status

blood vary depending on stress and correlate with the levels of cortisol in saliva (Kirschbaum \& Hellhammer, 1994).

Photometric method was used for the determination of cortisol in saliva. Cortisol has a diurnal rhythm: under normal conditions the level of cortisol increases 1-2 hours before waking up and reduces during the day until it reaches the minimum at midnight. Laboratory "E. Gulbja Laboratorija” has determined the following reference ranges of salivary cortisol: from 2.30 p.m. -3.30 p.m. $<11.9 \mathrm{nmol} / \mathrm{L}$. Samples were taken at 3.00 p.m. -3.30 p.m. The total amount of antioxidants was determined by the photometric method in heparinized blood plasma. In normal, antioxidant levels in a healthy person range from 1.3 to 1.77 $\mathrm{nmol} / \mathrm{L}$. All tests were carried out in an accredited laboratory, which performed testing in accordance with the international standard (LVS EN ISO 15189: 2008).

Before and after each massage session the study participants were filmed, all video - analysed, using the FaceReader $6.0^{\mathrm{TM}}$ software to perform an analysis of their emotional condition and define which of the six basic feelings (happy, angry, sad, surprised, frightened, disgust or neutral) prevail (Ekman \& Cordaro, 2011). In the study FaceReader 6.0 was used to test well-being by measuring the levels of positive and negative feelings. Video analysis and results mathematical statistics were performed Noldus Information Technology BV (Netherlands), www.noldus.com.

The study analysed BP parameters, particularly changes in systolic and diastolic pressure, and their progression to normal intervals. BP measurements were performed before each massage using the automatic BP measurement apparatus Omron M7 - IT. A remeasurement on the same arm was performed after the massage.

Back massage was performed in accordance with the following principles:

- positioning of the customer according to his individual needs before starting a massage;

- $\quad$ massage duration - 25 minutes;

- $\quad$ massage is performed for the back part from C7 to S5;

- massage is performed using the following sequence of techniques: gliding, rubbing, kneading and vibration.

Statistical analysis was performed using the statistical software package SPSS 17.0 for Windows. Data were expressed as mean and standard deviation. The differences in the distribution of qualitative variables were assessed by the Chi-square test. The difference considered to be significant when $\mathrm{p}<0.05$. Regression testing was performed by using the Fisher criterion. 


\section{Research results}

The results of the study showed no significant differences between the two groups $(\mathrm{p}<0.01)$ (Table 1). Repeated analysis of total blood scenes and body composition allowed an assessment of the effects of the procedure. The analyses were conducted four times during the study. The results showed that the subjects were generally healthy, and therefore the results were not analysed.

Table 1 Descriptive statistics of the anthropometry of the A group and B group

\begin{tabular}{|c|c|c|c|c|}
\hline & & & Mann-Whitney test for $\mathrm{p}=0.01$ and $\mathrm{n}=15$ \\
\hline Parameters & A group & B group & $\mathrm{U}$ & $\mathrm{U}_{\text {krit }}$ \\
\hline Age (years) & $25.0 \pm 5.3$ & $23.7 \pm 5.3$ & 86 & 51 \\
\hline Weight $(\mathrm{kg})$ & $63.5 \pm 9.0$ & $65.2 \pm 7.6$ & 79 & 51 \\
\hline Height $(\mathrm{m})$ & $170.7 \pm 6.6$ & $169.5 \pm 8.4$ & 87 & 51 \\
\hline BMI $(\mathrm{kg} / \mathrm{m} 2)$ & $21.7 \pm 2.2$ & $22.6 \pm 1.2$ & 84 & 51 \\
\hline \multicolumn{5}{|l|}{ BMI=body mass index. Values are expressed as mean \pm standart deviation $\mathrm{n}=15$} \\
\hline
\end{tabular}

Before intervention both groups were almost similar in regard to total antioxidant capacity, salivary cortisol level and BP levels, with the exception, that the salivary cortisol level was slightly higher for group A; in any case - all rates were within normal limits and all differences between both groups were statistically insignificant ( $>0.05$ ) (Table 2).

Table 2 Descriptive statistics of the Cumulative Change of the A group and B group

\begin{tabular}{|c|c|c|c|c|c|c|}
\hline & & & $\begin{array}{c}\text { Before } \\
\text { massage } \\
\text { course }\end{array}$ & $\begin{array}{c}\text { After the 5th } \\
\text { massage } \\
\text { session }\end{array}$ & $\begin{array}{l}\text { After the 10th } \\
\text { massage } \\
\text { session }\end{array}$ & $\begin{array}{c}\text { Two weeks } \\
\text { after massage } \\
\text { course }\end{array}$ \\
\hline \multirow{4}{*}{$\begin{array}{c}\text { Total } \\
\text { antioxidant }\end{array}$} & $\mathrm{A}$ & Mean & 1.58 & 1.37 & 1.61 & 1.63 \\
\hline & & SD & 0.13 & 0.13 & 0.13 & 0.12 \\
\hline & $\mathrm{B}$ & Mean & 1.59 & 1.56 & 1.76 & 1.63 \\
\hline & & SD & 0.09 & 0.13 & 0.05 & 0.08 \\
\hline \multirow{4}{*}{$\begin{array}{c}\text { Salivary } \\
\text { cortisol }\end{array}$} & $\mathrm{A}$ & Mean & 16.07 & 11 & 14.8 & 14.27 \\
\hline & & SD & 10.5 & 4 & 6.53 & 5.46 \\
\hline & $\mathrm{B}$ & Mean & 13.57 & 10.21 & 10 & 15.21 \\
\hline & & SD & 5.73 & 2.75 & 3.4 & 5.67 \\
\hline \multirow{4}{*}{$\begin{array}{c}\text { Systolic } \\
\text { BP } \\
\text { (mmHg) }\end{array}$} & $\mathrm{A}$ & Mean & 120.27 & 117.73 & 119.8 & 121.27 \\
\hline & & SD & 5.56 & 6.5 & 5.09 & 7.1 \\
\hline & $\mathrm{B}$ & Mean & 119.27 & 116.87 & 116.6 & 117.93 \\
\hline & & SD & 7.74 & 6.82 & 6.81 & 7.53 \\
\hline \multirow{4}{*}{$\begin{array}{l}\text { Diastolic } \\
\text { BP } \\
(\mathrm{mmHg})\end{array}$} & $\mathrm{A}$ & Mean & 73.47 & 71.33 & 72.47 & 73.07 \\
\hline & & SD & 4.91 & 5.02 & 4.03 & 6.1 \\
\hline & $\mathrm{B}$ & Mean & 72.8 & 69.4 & 72.47 & 70.73 \\
\hline & & SD & 4.31 & 5.79 & 5.1 & 5.97 \\
\hline
\end{tabular}


Veseta et al., 2020. Significance of Intensity of Swedish Massage Course in Improvement of Health Status

Salivary cortisol levels, decreased after the first five massage sessions in both groups, but the data are not statistically significant ( $p>0.05)$. The effects of Swedish massage on the physiological and biochemical factors of healthy individuals were irrelevant, excluding heart rate.

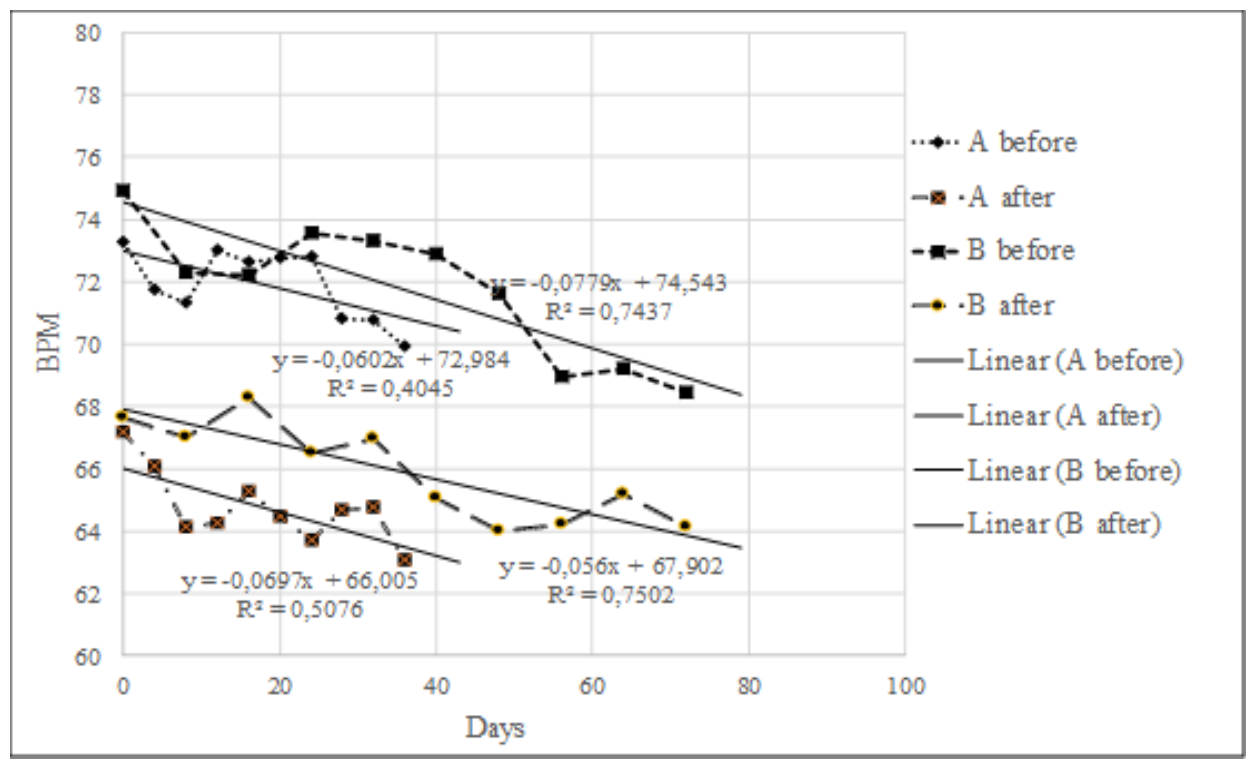

Figure 2 Heart rate of the A group and B group

Before intervention, the heart rates for group A and B did not differ significantly $(\mathrm{p}>0.05)$. Slope position was determined by Fisher criterion. With a $5 \%$ reduction in probability will be all the time. Statistically significant $(\mathrm{p}<0.05)$ (Figure 2, Table 3).

Table 3 Descriptive statistics of the heart rate of the A group and B group

\begin{tabular}{|c|c|c|c|c|c|c|}
\hline & & & \multicolumn{4}{|c|}{ For Slope } \\
\hline & $\begin{array}{c}\text { Interce } \\
\mathrm{pt}\end{array}$ & Slope & $F$ & Significance F & Lower 95.0\% & Upper 95.0\% \\
\hline A before & 72.984 & -0.0602 & 5.43 & 0.04808 & -0.1198 & -0.000647 \\
\hline A after & 66.005 & -0.0697 & 8.25 & 0.02078 & -0.1257 & -0.013728 \\
\hline B before & 74.543 & -0.0779 & $\begin{array}{c}23.2 \\
1\end{array}$ & 0.00132 & -0.1152 & -0.040633 \\
\hline B after & 67.902 & -0.0560 & $\begin{array}{c}24.0 \\
2\end{array}$ & 0.00119 & -0.0823 & -0.029635 \\
\hline
\end{tabular}

The students' independent t-test results showed that the average score of neutral and happy emotions was different between the A and B groups before and after intervention ( $>>0.05)$ (Figure 3). 


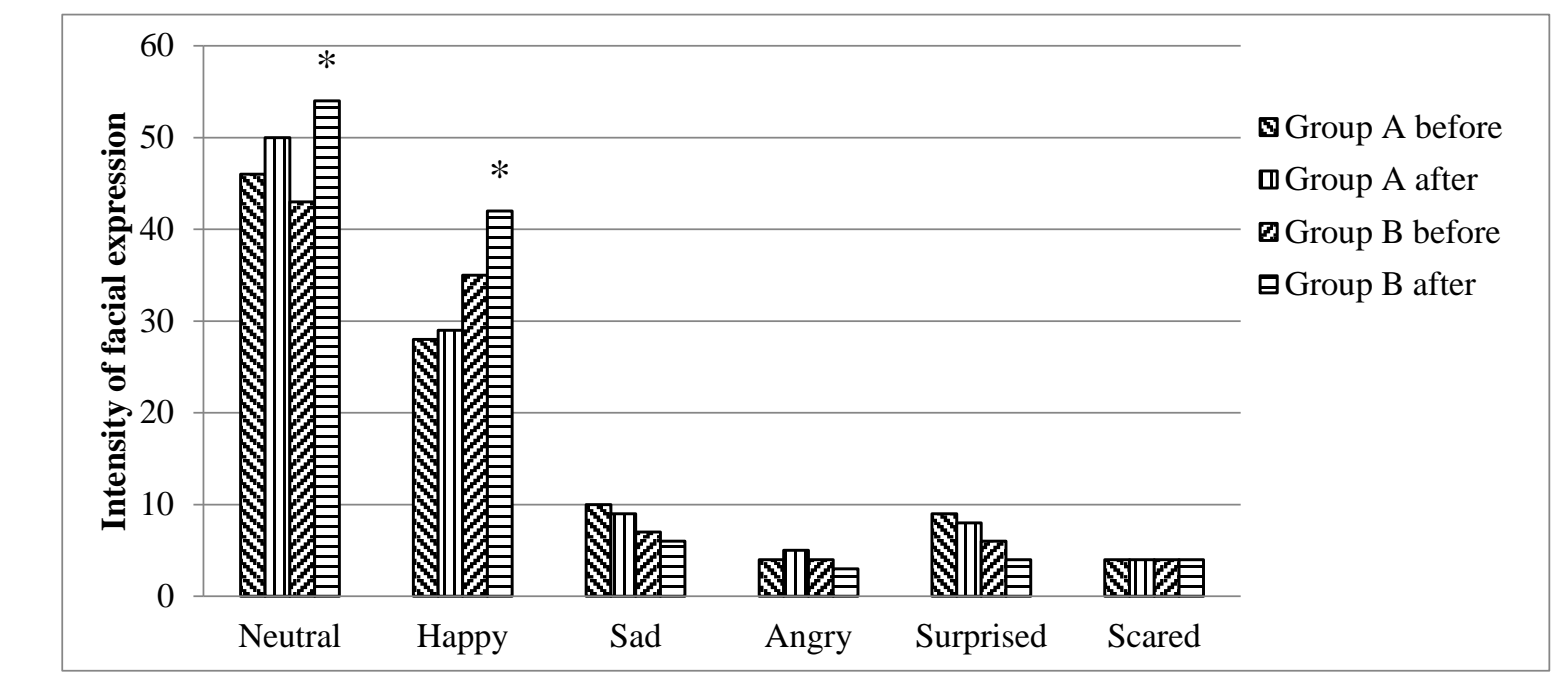

* significant differences compared to before massage course $(p \leq 0.05)$

Figure 3 Induced emotions before and after massage of the A group and B group

\section{Conclusions and discussion}

This was a 5 and 10 weeks randomized open-type comparative study that compared the immediate and cumulative effects of Swedish massage to the physiological, emotional and biochemical factors of healthy individuals, as well as the sustainability of massage impact depending on the intensity of the course. Blood pressure, pulse and emotion measurements were performed before and after each massage session to determine the immediate effects of massage. The results of the study showed that there were no significant differences between the two groups $(\mathrm{p}<0.01)$, while average physiological parameters were close to the upper limit of norm. The study data showed that the effect of a single massage was weak, similar to that of Rapaport, Schettler, \& Breese (2010) study. The impact analysis of each massage session showed minor changes in determinations of physiological and emotional factors, but before each following massage there were positive determination trends.

The analysis of these determinants of group A (a massage course twice a week) showed higher fluctuations in results, which generally ensured less stabilization of physiological and emotional factors. Data of group B (a massage course once a week) showed higher stabilizing effects of the results. The proportion of neutral and happy feelings increased during the course and heart rate decreased, which is statistically significant $(\mathrm{p}<0.05)$.

While massage therapy lacks evidence-based research, its popularity is rising. It's important to consider the availability of massage. Massage is performed twice a week in most studies (Fielda, 2016). For example, the study of Fateme, Mojtaba, \& Hojatollah (2015) demonstrated the importance of twice a week massage in health maintenance and in work quality of intensive therapy units' 
Veseta et al., 2020. Significance of Intensity of Swedish Massage Course in Improvement of Health Status

nurses. Similar studies analysing the effect's sustainability would be required for massage course with sessions performed once a week.

Another study (Sharpe, Williams, Granner, \& Hussey, 2007) shows that massage therapy improves positive well-being and reduces stress perception among older adults. It would be necessary to carry out once per week massage session studies for this target group in order to consider the possibility to ensure broader availability of massage. As mentioned above, our study concluded that in practically healthy young adults, massage provided on once per week basis increased positive feelings during a massage course $(\mathrm{p}<0.05)$.

As in the Field et al. (1996) study, the results of this study showed a tendency to impact the levels of saliva cortisol. Saliva cortisol levels decreased after the first five massage sessions in both groups, but the data is not statistically significant ( $>00.05)$. Saliva cortisol level is a good indicator for analysing the immediate effects of Swedish massage.

Givi (2013) and Moeini, Givi, Ghasempour, \& Sadeghi (2011) examine the effect of massage on blood pressure. The study was conducted three times a week in women with pre-hypertension. In our study participated healthy subjects, and the effect of massage on blood pressure is not statistically significant ( $p>0.05$ ). Nor did the study confirm a significant increase in total antioxidant levels.

Comparing heart-rate scores in both groups heart rates declined regardless of whether massage was performed two or once per week. This expected finding is supported by earlier studies (Garner et al., 2008) as well.

Based on the findings of this study, it may be concluded that a once per week massage course is recommended to improve the health status of practically healthy people. Similar studies in older people and people with increased levels of workplace stress would be recommended in future.

\section{References}

Bunker, S.J., Colquhoun, D.M., Esler, M.D., Hickie, I.B., Hunt, D., Jelinek, V.M. et al. (2003). "Stress" and coronary heart disease: Psychosocial risk factors. Medical Journal of Australia, 178, 272- 276.

Dickerson, S.S., \& Kemeny, M.E. (2004). Acute stressors and cortisol responses: a theoretical integration and synthesis of laboratory research. Psychologican Bulletin, 130, 355-391. DOI:10.1037/0033-2909.130.3.355

Ekman, P., \& Cordaro, D. (2011). What is meant by calling emotions basic. Emotion review, 3(4), 364-370. DOI:10.1177/1754073911410740

Fateme, N., Mojtaba, M., \& Hojatollah, Y. (2015). The effect of massage therapy on occupational stress of Intensive Care Unit nurses. Iranian journal of nursing and midwifery research, 20(4). DOI:10.4103/1735-9066.161001

Field, T. (2016). Massage therapy research review. Complementary Therapies in Clinical Practice, 24. DOI: 10.1016/j.ctcp.2016.04.005

Field, T., Hernandez-Reif, M., Diego, M., Schanberg, S., \& Kuhn, C. (2005). Cortisol 
Decreases and Serotonin and Dopamine Increase Following Massage Therapy. International Journal of Neuroscience, 115, 1397-1413. DOI: 10.1080/ 00207450590956459

Field, T., Ironson, G., Scafidi, F., Nawrocki, T., Goncalves, A., Burman, I. et al. (1996). Massage Therapy Reduces Anxiety and Enhances Eeg Pattern of Alertness and Math Computations. International Journal of Neuroscience, 86, 197-205. DOI: 10.3109/00207459608986710

Fritz, S. (2014). Mosby’s Massage Therapy Review. 4th ed. Mosby.

Garner, B., Phillips, L.J., Schmidt, H.M., Markulev, C., O'Connor, J., Wood, S.J., Berger, G.E., Burnett, P., \& McGorry, P.D. (2008). Pilot study evaluating the effect of massage therapy on stress, anxiety and aggression in a young adult psychiatric inpatient unit. Australian \& New Zealand Journal of Psychiatry, 42(5), 414 - 422.

Givi, M. (2013). Durability of Effect of Massage Therapy on Blood Pressure. International Journal of preventive medicine, 4(5), 511- 516. [Data file]. Retrieved from https://www.ncbi.nlm.nih.gov/pmc/articles/PMC3733180/

Kirschbaum, C., \& Hellhammer, D.H. (1994). Salivary cortisol in psychoneuroendocrine research: Recent developments and applications. Psychoneuroendocrinology, 19(4), 313333. DOI: 10.1016/0306-4530(94)90013-2

Moeini, M., Givi, M., Ghasempour, Z., \& Sadeghi, M. (2011). The effect of massage therapy on blood pressure of women with pre-hypertension. Iranian journal of nursing and midwifery research, 16(1), 61-70. [Data file]. Retrieved from https://www.ncbi.nlm. nih.gov/pubmed/22039381

Moraska, A., Pollini, R.A., Boulanger, K., Brooks, M.Z., \& Teitlebaum, L. (2010). Physiological adjustments to stress measures following massage therapy: a review of the literature. Evidence-Based Complementary and Alternative Medicine, 7(4), 409- 418. DOI:10.1093/ecam/nen029.

Moyer, C.A., Rounds, J., \& Hannum, J.W. (2004). A Meta-Analysis of Massage Therapy Research. Psychologican Bulletin, 130, 3-18. DOI:10.1037/0033-2909.130.1.3.

Nakano, H., Kodama, T., Ueda, T., Mori, I., Tani, T., \& Murata, S. (2019). Effect of Hand and Foot Massage Therapy on Psychological Factors and EEG Activity in Elderly People Requiring Long-Term Care: A Randomized Cross-Over Study. Brain Sciences, 9(3), 54. DOI: $10.3390 /$ brainsci9030054

Rapaport, M.H., Schettler, P., \& Breese, C. (2010). A preliminary study of the effects of a single session of Swedish massage on hypothalamic-pituitary-adrenal and immune function in normal individuals. Journal of Alternative and Complementary Medicine, 16, 1079-1088. DOI: 10.1089/acm.2009.0634

Rapaport, M.H., Schettler, P., \& Bresee, C. (2012). A preliminary study of the effects of repeated massage on hypothalamic-pituitary-adrenal and immune function in healthy individuals: a study of mechanisms of action and dosage. Journal of Alternative and Complementary Medicine, 18, 789-797. DOI:10.1089/acm.2011.0071

Sharpe, P.A., Williams, H.G., Granner, M.L., \& Hussey, J.R. (2007). A randomised study of the effects of massage therapy compared to guided relaxation on well-being and stress perception among older adults. Complementary Therapies in Medicine, 15(3), 157-163. DOI: 10.1016/j.ctim.2007.01.004 